\title{
Numerical analysis of magnetic states mixing in the Heisenberg model with the dihedral symmetry
}

\author{
W. Florek ${ }^{1, a}$, L.A. Kaliszan ${ }^{2}$, K. Jaśniewicz-Pacer ${ }^{1}$, and M. Antkowiak ${ }^{1}$ \\ 1 Faculty of Physics, A. Mickiewicz University, Poznań, Poland \\ 2 Poznan School of Logistics, Poznań, Poland
}

\begin{abstract}
The total spin number $S$ is not a 'good quantum number for' the Heisenberg model with singleion anisotropy, so the Hamiltonian eigenstates with different $S$ may form linear combinations. Sometimes it is assumed that $S$ can be used as an 'approximate quantum number', though some results show that mixing of $S$ states is important in investigations of magnetic molecules. Some small spin systems with the dihedral symmetry are analyzed to investigate different schemes of mixing and its dependence on the anisotropy parameter. The results show various behavior of the magnetic state mixing. The mean (over a state) value of total spin is quite stable for the ground state, but in other cases this dependence is nonlinear and sometimes non-monotonic.
\end{abstract}

\section{Introduction}

Investigation of molecules containing magnetic centers is one of the most important topics in contemporary physics and chemistry [1]. Such research is motivated by possible applications of single-molecule magnets (SMM) as data storage [2] and building blocks of quantum computers [3, 4]. Two basic conditions have to be satisfied by a molecule to be an SMM: i) a high-spin ground state (GS) and ii) a large zero-field splitting [5]. The latter one is mainly caused by magnetic anisotropy resulting from the singleion anisotropies determined by organic ligands, their geometry near the metal centers etc. The single-ion anisotropy terms do not commute with the square of $\mathbf{S}=\sum_{j} \mathbf{s}_{j}$, so the total spin number cannot be used as an additional label of states or levels. In the other words: Since states do not need to have fixed the total spin number $S$, then they may form linear combinations of states with determined $S$. Sometimes it is assumed that for small anisotropy this 'mixing' can be neglected and $S$ can still be used as an 'approximated good quantum number'. There are some papers in which mean value of $S$ has been discussed (e.g. [6,7]), and the results presented have shown that such approach is not admissible in a general case. The aim of this work is to investigate eigenstates of the Heisenberg Hamiltonian containing the single-ion anisotropy term, especially a dependence of $\left\langle\mathbf{S}^{2}\right\rangle$ on the anisotropy parameter.

\section{Systems considered}

The following, very simple, model

$$
\mathcal{H}=\sum_{j=1}^{n} \mathbf{s}_{j} \cdot \mathbf{s}_{j+1}+\delta\left(s_{j}^{z}\right)^{2}
$$

is considered for even $n \leq 10$ and the spin number $1 \leq$ $s \leq 5 / 2$ (not all possible cases are discussed, but for $n=2$

\footnotetext{
a e-mail: wojciech.florek@amu.edu.pl
}

the spin number $s \leq 20$ ); the anisotropy parameter $\delta$ is expressed in units of the exchange integral. This form suggests antiferromagnetic coupling, but the results can be applied to both, ferro- (FM) and antiferromagnetic (AFM) systems, since energies are irrelevant. At first the eigenvectors $\left|S r_{S}\right\rangle$ of $\mathbf{S}^{2}$ are determined (the index $r_{S}$ distinguishes states with the same $S$ ). In this basis the eigenproblem for the Hamiltonian (1) is solved, so the eigenvectors

$$
\left|E r_{E}\right\rangle=\sum_{S} \sum_{r_{S}} \alpha\left(E, r_{E} ; S, r_{S}\right)\left|S r_{S}\right\rangle
$$

are determined ( $r_{E}$ is a repetition index in degenerated energy levels). The mean value of $\mathbf{S}^{2}$ is, of course,

$$
\left\langle\mathbf{S}^{2}\right\rangle_{E, r_{E}}=\sum_{S} S(S+1) \sum_{r_{S}}\left|\alpha\left(E, r_{E} ; S, r_{S}\right)\right|^{2} .
$$

The symmetry group of the Hamiltonian (1) is isomorphic with the dihedral group $\mathrm{D}_{n}$ and its irreducible representations (irreps) are used as a part of repetition indices $r_{S(E)}$. Moreover, $\mathcal{H}$ commutes with $\sum_{j} s_{j}^{z}$, so the above procedure can be performed for each value of the magnetization $M,|M| \leq n s$, independently. Due to the time reversal (TR) symmetry it is enough to consider $M \geq 0$. For $M=0$ states related by the TR operator have the same $M$ and geometrical symmetry (labeled by irreps of $\mathrm{D}_{n}$ ), so their superpositions symmetric (gerade) and antisymmetric (ungerade) with respect to the TR can be constructed. To facilitate calculations some combinatorial aspects of the problem can be taken into account (cf. [8]).

\section{Results}

In this section only some, most important, cases are presented, but it suffices to gain better understanding of the eigenstates and its properties. To reduce size of figures the mean value $\bar{S}(\delta)$ is plotted, where $\bar{S}$ is a non-negative root of $\bar{S}(\bar{S}+1)=\left\langle\mathbf{S}^{2}\right\rangle$. The considerations are limited to fully

This is an Open Access article distributed under the terms of the Creative Commons Attribution License 2.0, which permits unrestricted use, distribution, and reproduction in any medium, provided the original work is properly cited. 


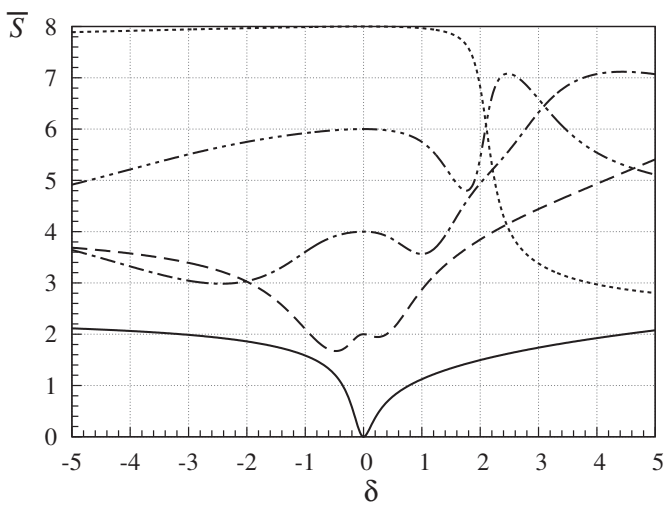

Fig. 1. The mean value $\bar{S}$ for pair of spins $s=4$.

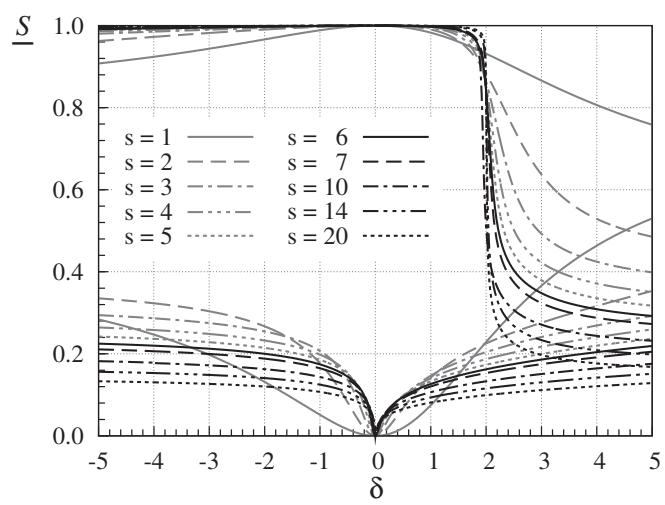

Fig. 2. The normalized mean value $\underline{S}$ in the AFM and FM GS's for pair of spins $s=1,2,4,6,10,20$.

symmetric states with $M=0$. Note that for $\delta=0$ the GS's of AFM and FM systems systems belong to this subspace if $n s$ is even. For $\delta \neq 0$ this statement is still valid for the AFM GS, but states with $M \neq 0$ can be the FM GS in this case (especially for $\delta<0$ ). In this paper the FM GS denotes the state with the largest eigenvalue of (1) and $S=n s$ for $\delta=0$.

\subsection{Pair of spins}

The results for $s=4$ are presented in figure 1, where the characteristic dependencies $\bar{S}(\delta)$ can be observed. The AFM GS $(\bar{S}(0)=0)$ shows the V-shape near $\delta=0$, but for the excited states $(\bar{S}(0)=2,4)$ the $\mathrm{W}$-shape is rather seen. This shape is also visible for all states with $\bar{S}(0) \neq 0,2 s$, but the states near the FM GS have also rapid changes of $\bar{S}$ near $\delta=2$. At this point the value of $\bar{S}$ for the FM GS decreases very fast, whereas it is quite stable for $\delta<0$. To compare the results for different spin numbers a normalized value $\underline{S}=\bar{S} / 2 s$ is introduced (so $\underline{S}(0)=1$ in the FM GS) and it is plotted in figure 2. For small $\delta$ the value of $S$ is quite stable in the FM GS and it is even more stable for larger spin numbers $s$. For the AFM GS this dependence is reversed. For example, when $\delta=0.1$ this value grows from 0.001 for $s=1$ to 0.041 for $s=10$. For $|\delta| \rightarrow \infty$ the mixing of states with a determined total spin number $S$ is dominant for $s=1$ (except $\delta \rightarrow \infty$ and the FM GS); for

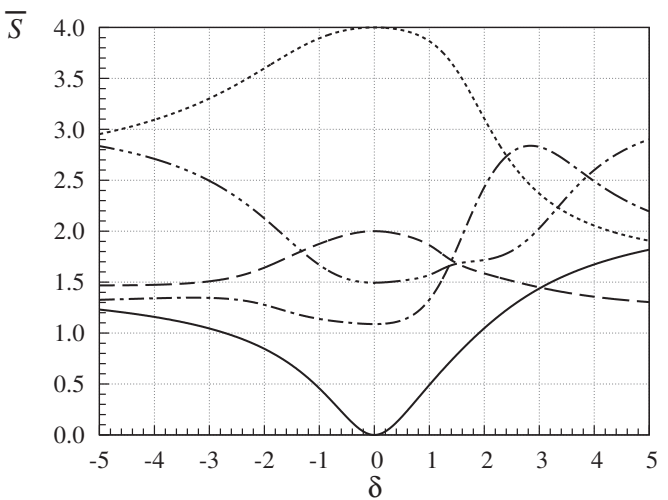

Fig. 3. The mean value $\bar{S}$ for four spins $s=1$.

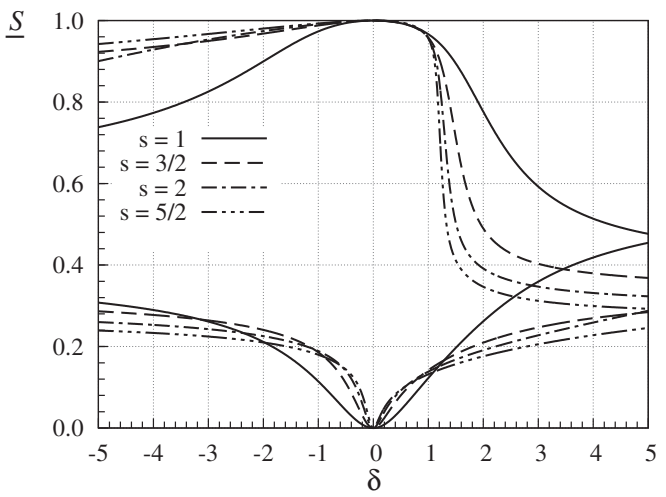

Fig. 4. The normalized mean value $S$ in the AFM and FM GS's for four spins $s=1,3 / 2,2,5 / 2$.

$s=1$ and the AFM GS it can be shown that $\underline{S}(\delta) \rightarrow 0.5$ (exactly) for $\delta \rightarrow-\infty$ and to about 0.781 for $\delta \rightarrow \infty$.

\subsection{Four spins}

Considering spin pairs it suffices to take into account properties of the step operators $s^{ \pm}$and with some algebra one obtains general formulas. For larger spin systems it is convenient to apply group theoretical and combinatorial methods [8]. Even for $n=4$ such preliminary analysis is helpful. For example, when $s=5 / 2$ there are 146 states with $M=0$ and solving the eigenproblem is not difficult. However, most of algorithms do not preserve the order of energy levels when this problem is solved for different values of the anisotropy parameter (the Jacobi algorithm [9] has been used in our calculations). Group-theoretical classification of states limits possible superpositions to states with the same symmetry properties and corresponding to the same values of good quantum numbers (the magnetization $M$ in the problem discussed). From the above mentioned 146 states one can obtain 25 fully symmetric (with respect to the symmetry group $\mathrm{D}_{4}$ ) linear combinations. The TR symmetry yields further decomposition: twenty of them are gerade and only five superpositions are ungerade.

The system of four spins $s=1$ was discussed in our previous paper [10] (without the TR symmetry). The results for five fully symmetric (and gerade) states is presented in figure 3. For $\delta=0$ two states, with $S=0,2$, have the same energy, so any linear combination of them 

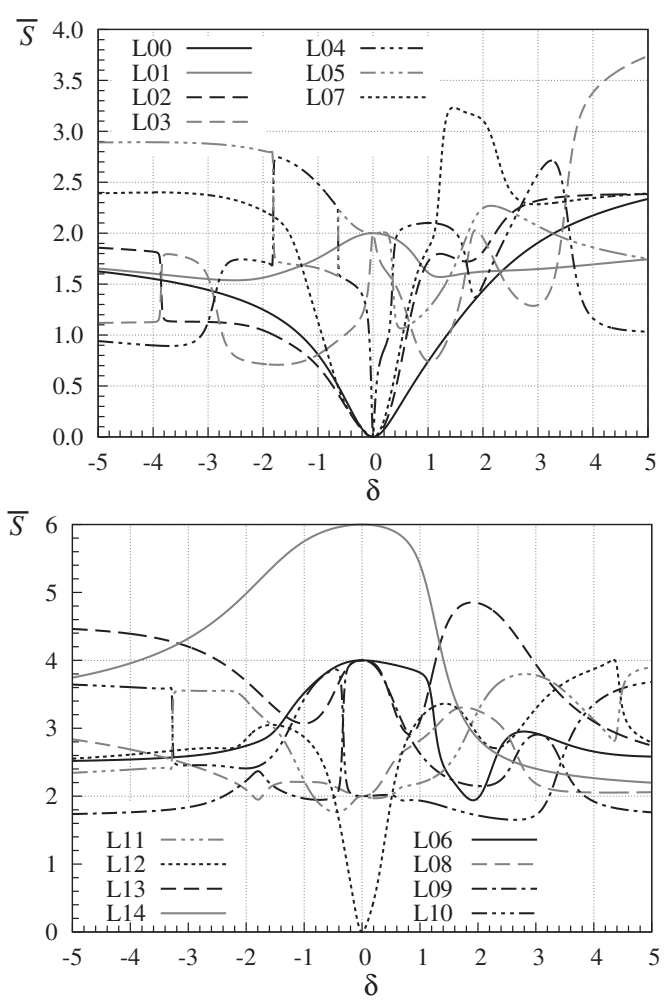

Fig. 5. The mean value $\bar{S}$ for six spins $s=1$.

is the Hamiltonian eigenstate and, in fact, $\bar{S}$ is not determined at this point. However, for $|\delta| \rightarrow 0$ we have obtained $\bar{S} \rightarrow 1.09$ and $1.49\left(\left\langle\mathbf{S}^{2}\right\rangle \approx 2.28\right.$ and 3.71, respectively). The energy degeneration of these two states destroys the W-shape of the appropriate plots, but such behavior is observed for larger spin numbers. Note that the AFM GS has V-shape plot near $\delta=0$; the FM GS is not so stable as for $n=2$ and $\bar{S}(\delta)$ decreases faster for $\delta \approx 1.0$. It is worth recalling one of the results from the previous paper [10]. For $M=1$ there are three states with $B_{1}$ symmetry and $S=1,2,3$ for $\delta=0$. For any $\delta \neq 0$ states with $S=2,3$ form a linear combinations with coefficients independent on $\delta$ and $\left\langle\mathbf{S}^{2}\right\rangle=9 \frac{1}{3}$.

The similar calculations have been performed also for $s=3 / 2,2,5 / 2$ and the behavior of $S(\delta)$ for the GS's is presented in figure 4. Qualitatively this diagram is analogous as for spin pairs, but the rapid drop of $S(\delta)$ for the FM GS is observed near $\delta=1$.

\subsection{Rings with $s=1$}

The rings with $n=6,8,10$ spins $s=1$ have been considered to observe changes in $\bar{S}$ with increasing system size. For $n=2,4$ there are no states antisymmetric under the TR operation. A number of such states grows rapidly and for $n=10$ there are 290 symmetric and 196 antisymmetric states $(M=0$ and states are symmetric with respect to $\left.\mathrm{D}_{10}\right)$. For $n=12$ these numbers are 1728 and 1451, respectively, so it can be predicted that for larger $n$ these numbers are almost equal (there are always more gerade states).

For $n=6$ there are only three ungerade states and, moreover, two of them are degenerated with the energy
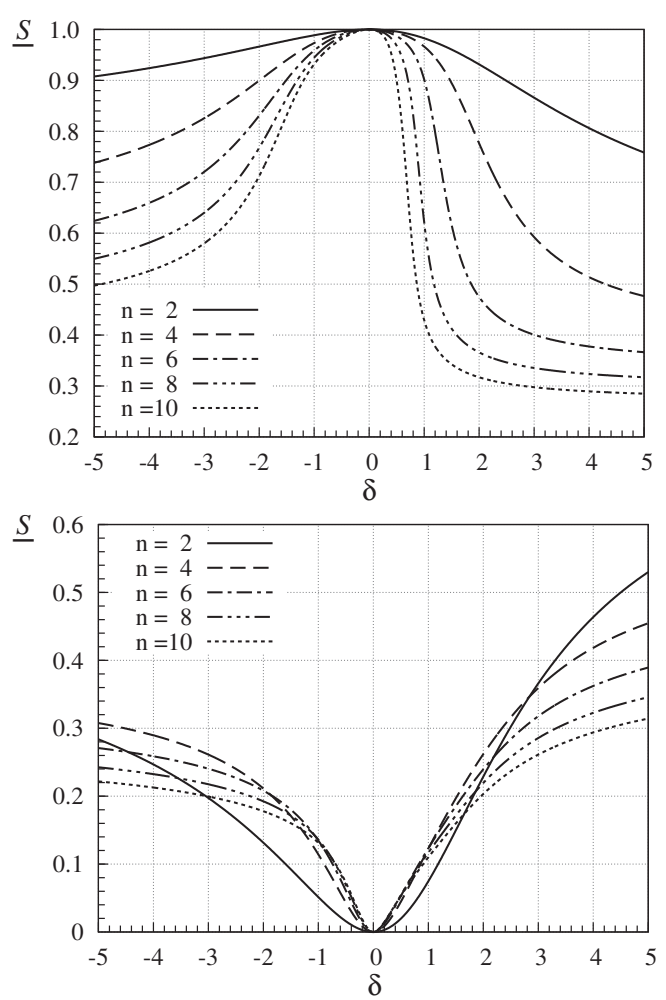

Fig. 6. The normalized mean value $\underline{S}$ for $2 \leq n \leq 10$ spins $s=1$.

$4 \delta-2$. They correspond to $S=1,3$ but, due to the degeneracy, any value $2 \leq \bar{S} \leq 3$ can be obtained. The third state has $S=3$ and it does not enter any linear combination, so it preserves this value for any $\delta$. In general, at least in these simple cases considered here, the ungerade states are related to odd total spin numbers $S$. Since the GS's of FM and AFM systems are mainly discussed, so the antisymmetric states will not be considered hereafter.

There are 15 states with full geometrical and TR symmetry. For $\delta=0$ they correspond to $S=0,2,4,6$ and numbers of each $S$-multiplets are, respectively, as follows: $5,6,3,1$. To make pictures more clear they are decomposed into two groups of levels: A) $0-4,5,7$ and B) 6, 8-14. Each level is denoted as $\mathrm{L} x$, with $0 \leq x \leq 14$, where the numbers $x$ corresponds to the energy levels order (L00 denotes the AFM GS, so L14 is the FM GS). The plots of $\bar{S}(\delta)$ are presented in figure 5. All states with $S=0$ for $\delta=0$ (L00, L02, L03, L04, and L12) have V-shape plots near $\delta=0$. Especially for the level L04 changes in $\bar{S}$ are very fast. The W-shape plots, observed for $n=2$, are still visible for the levels L01, L11, and L13. The FM GS is well separated from the others and shows rapid change in $\bar{S}$ about $\delta=1$. The plots for other levels are very irregular and there are a few points at which two levels interchanges their values of $\bar{S}$. It can be seen for the levels L03 and L04 near $\delta=-3,2$ or 4 . These changes are very rapid in some cases (the levels L02, L03 near $\delta=-4$ or the levels L04 and L05 near $\delta=-1$ and -0.5$)$, and we have carefully checked these points to exclude artifacts caused by numerical algorithms. The energy levels are very close for these values of $\delta$, but energy levels never cross each other. For example, energies for the levels L04 and L05 are about -4.982 and -4.979 at $\delta=-0.634$, so it is sufficient accuracy to say that these levels do not cross. 


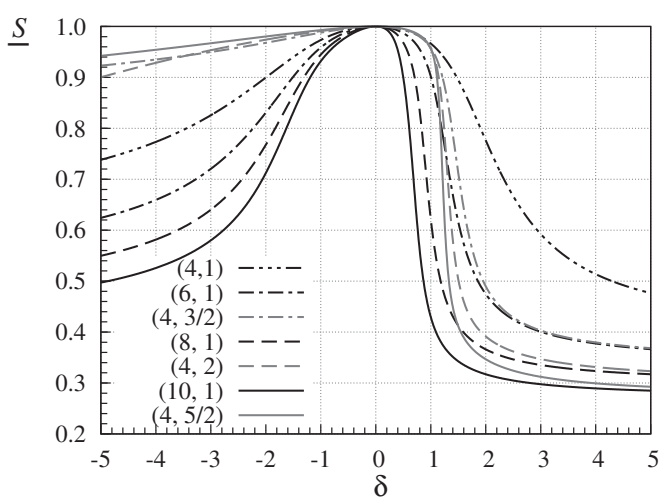

Fig. 7. The normalized mean value $\underline{S}$ in the FM GS for different pairs $(n, s)$.

As in the previous cases the normalized mean values $\underline{S}$ are compared in figure 6 (the previous results for $n=$ $\frac{5}{2}, 4$ are included). For larger systems with $s=1$ the FM GS changes its $\left\langle\mathbf{S}^{2}\right\rangle$ more and more rapidly. The inflexion points for $\delta>0$ plotted vs. $1 / n$ gives a straight line with an intercept near 0 , but with so few samples this result cannot be considered as well-funded. The same calculations for $\delta<0$ yield the limit $c a 1.5$ for $1 / n \rightarrow 0$. For the AFM GS changes in the plots of $S(\delta)$ are not so distinct (except for the difference between plots for $n=2$ and $n=4)$. For example, $S(\delta)=0.1$ for $\delta \approx 0.85$ (for $n=4,6,8,10$ ) and for $\delta$ between $-0.68(n=10)$ and $-0.9(n=4)$. Looking from the other side, one can say that the total spin is quite stable in the AFM GS, since for $|\delta| \leq 0.1$ the normalized mean value is less than 0.005 .

\subsection{Final remarks}

The changes in the value of $\underline{S}$ discussed above show that increasing both the spin number $s$ and the system size leads to more intense modifications in the plots. In figure 7 all results obtained for the FM GS are presented, but lines are labeled by the pairs $(n, s)$ (results for $n=2$ are excluded). It seems that $\lim _{\delta \rightarrow \infty} \underline{S}(\delta)$ depends only on the product $n s$. However, for finite values of $\delta$ the size of a system leads to more visible changes in $S$ than the spin number. It is especially clear for $\delta<0$, where plots for four spins $s=$ $3 / 2,2,5 / 2$ are almost identical.

\section{Conclusions}

At first note that the magnetic states shows full spectrum of different mixing schemes. Some states preserves its total spin number $S$ even in cases, when there are other states with the same magnetization and symmetry properties. In other cases one can observe the mixing with $\left\langle\mathbf{S}^{2}\right\rangle$ independent on $\delta$. When states are degenerated for $\delta=0$ (as for four spins $s=1$ ) then any linear combination is the Hamiltonian eigenstate, but they form a linear combination with nontrivial and relatively large coefficients for any $\delta \neq 0$ (in the above mentioned case squares of these coefficients are about 0.38 and 0.62 ). On the other hand the levels L03 and L04 are nondegenerated at $\delta=0$, so with $\delta \rightarrow 0$ they have to be the eigenstates of $\left\langle\mathbf{S}^{2}\right\rangle$ and they do it really fast: numerically calculated derivative of $\bar{S}(\delta)$ for L04 is about -20 at $\delta=-0.02$ and $\left\langle\mathbf{S}^{2}\right\rangle$ is about 0.29 at this point. In some states for all values of $\delta$ one state is dominant and coefficients of other $S$-states are relatively small, so $\left\langle\mathbf{S}^{2}\right\rangle$ is close to its value at $\delta=0$. However, in general cases, a group of states have coefficients with comparable magnitudes. It is obvious (and can be easy shown) that mixing is more intense when energy levels are close, so, for example, the AFM GS, is mainly a superposition of states with $S=0$ and $S=2$ for small $\delta$, despite the fact that there are some other states with $S=0$ at $\delta=0$.

The presented results shows that the mean value of $\mathbf{S}^{2}$ is quite stable in the GS's, since they are well-separated in quantum spin models of (anti)ferromagnetic magnetic molecules (there are many papers presenting the energy levels in such models). Therefore, in these cases using the 'approximate quantum number $S$ ' is acceptable ( $c f$. the end of Sect. 3.3). However, as we have shown, it cannot be extended to states with intermediate energies. Moreover, in all cases the changes in $\bar{S}$ are more intense for larger spin numbers $s$ (for example in molecules comprising rare earth ions) and larger systems. The rapid drop of $\underline{S}$ for the FM GS tends to have place at $\delta=4 / n$ for $s \rightarrow \infty$ (see figures 6 and 7), so for $n=20$ and large spin numbers it should happen just above, not so large, $\delta=0.2$. Hence, one should not use $S$ as a quantum number in such cases.

It is important that energy levels interchange the mean value of $\mathbf{S}^{2}$, when they are close (but nondegenerated). In larger systems it may happen for quite small values of $\delta$. E.g. plots of $\bar{S}(\delta)$ for the levels L09 and L10 (six spins $s=1$ ) cross each other at $\delta=0.33$ (see figure5). In the same figure one can see that this process can be very rapid: the levels L04 and L05 completely 'interchange' $\bar{S}(\delta)$ for $-0.64<\delta<-0.62$ and absolute values of the appropriate derivatives $\bar{S}^{\prime}(\delta)$ are about 100 .

\section{References}

1. D. Gatteschi, R. Sessoli, J. Villain, Molecular Nanomagnets (Oxford Univ. Press, Oxford 2006)

2. M. Mannini, F. Pineider et al., Nature Mater. 8, 194 (2009)

3. G.A. Timco, S. Carretta et al., Nature Nano. 4, 173 (2009)

4. B. Georgeot, F. Mila, Phys. Rev. Lett. 104, 200502 (2010)

5. J. van Slageren, R. Sessoli et al., Chem. Eur. J. 8, 277 (2002)

6. M. Affronte, T. Guidi et al., J. Mag. Mag. Mater. 272276, 1050 (2004)

7. S. Carretta, P. Santini et al., Phys. Rev. Lett. 98, 167401 (2007)

8. W. Florek, S. Bucikiewicz, Phys. Rev. B 66, 024411 (2002)

9. W.H. Press, S.A. Teukolsky, W.T. Vetterling, B.P. Flannery, Numerical Recipes in C, (Cambridge Univ. Press, Cambridge 1992)

10. W. Florek, K. Jaśniewicz-Pacer, L.A. Kaliszan, G. Kamieniarz, Cen. Eur. J. Chem. 7, 211 (2009). 